\title{
Potential of High Dimensional Radiomic Features to Assess Flowing Blood Components in Non-contrast CT Scans
}

\author{
Scherwin Mahmoudi \\ University Hospital Frankfurt \\ Simon S. Martin \\ University Hospital Frankfurt \\ Jörg Ackermann \\ Goethe University Frankfurt \\ Yauheniya Zhdanovich \\ Goethe University Frankfurt \\ Ina Koch \\ Goethe University Frankfurt \\ Thomas J. Vogl \\ University Hospital Frankfurt \\ Moritz H. Albrecht \\ University Hospital Frankfurt \\ Lukas Lenga \\ University Hospital Frankfurt \\ Simon Bernatz ( $\nabla$ simon.bernatz@kgu.de) \\ University Hospital Frankfurt
}

\section{Research Article}

Keywords: Radiomics, Blood, Anemia, Artificial Intelligence, CT

Posted Date: April 7th, 2021

DOl: https://doi.org/10.21203/rs.3.rs-390527/v1

License: @) (7) This work is licensed under a Creative Commons Attribution 4.0 International License. Read Full License

Version of Record: A version of this preprint was published at BMC Medical Imaging on August 12th, 2021. See the published version at https://doi.org/10.1186/s12880-021-00654-9. 


\section{Abstract}

Background: To assess the potential of radiomic features to quantify components of flowing blood to non-invasively predict moderate-to-severe anemia in non-contrast enhanced CT scans.

Methods: One hundred patients (median age, 69 years; range, 19-94 years) who received CT scans of the thoracolumbar spine and blood-testing for hemoglobin and hematocrit levels $\pm 24 \mathrm{~h}$ between $08 / 2018$ and $11 / 2019$ were retrospectively included. Intraaortic blood was segmented using a spherical volume of interest with consecutive radiomic analysis. Feature selection was performed applying analysis of correlation and collinearity. The final feature set was obtained to differentiate moderate-to-severe anemia. Random forest machine learning was applied and predictive performance was assessed. A decision-tree was obtained to propose a cut-off value of CT Hounsfield units (HU).

Results: High correlation with hemoglobin and hematocrit levels was shown for first-order radiomic features ( $p<0.001$ to $p=0.032)$. The top 3 features showed high correlation to hemoglobin values ( $p)$ and minimal collinearity $(r)$ to the top ranked feature Median $(p<0.001)$, Energy ( $p=0.002, r=0.387)$, Minimum $(p=0.032, r=0.437)$. Median ( $<0.001)$ and Minimum ( $p=0.003)$ differed in moderate-to-severe anemia compared to non-anemic state. Median yielded superiority to the combination of Median and Minimum $(\mathrm{p}(\mathrm{AUC})=0.015, \mathrm{p}($ precision $)=0.017, \mathrm{p}($ accuracy $)=0.612)$ in the predictive performance employing random forest analysis. A Median HU value $\leq 36.5$ indicated moderate-to-severe anemia (accuracy=0.90, precision=0.80).

Conclusions: First-order radiomic features correlate with hemoglobin levels and may be feasible for the prediction of moderate-to-severe anemia. High dimensional radiomic features did not inherit the potential to augment the data in our exemplary use case of flowing blood component assessment.

Trial registration: Retrospectively registered.

\section{Background}

Radiomics is a term coined for computational quantitative imaging analysis and has been shown to inherit the potential in aiding clinical decision making (1). Radiomics extracts a large number of quantitative data from medical images that can provide surrogate information on biochemical and pathophysiological processes $(2,3)$. The technique has been successfully applied to evaluate tumor characteristics non-invasively $(4)$. While several studies showed the benefits of radiomics in solid tissue and predominantly cancer research (5-7), its potential to assess flowing structures and moving tissues has not yet been investigated.

Acute and chronic blood loss might not only be surrogates of yet undiagnosed diseased which require further workup but also might be considered as an illness itself which requires haemostasis management $(8,9)$. In emergency patients with acute blood loss, fast assessment of a multitude of blood components, a.o. hemoglobin and hematocrit levels is essential $(10,11)$. In 2002, the World Health Organization has attributed anemia as one of the most relevant risk factors leading to high mortality and morbidity $(12,13)$. During hospitalization, phlebotomy is the current standard of screening for a load of blood components (14). Blood samples are usually easily obtained, but the procedure can be time consuming in some cases (15). Non-invasive screening of blood components in a clinically indicated CT may yield the potential to assess specific blood components in order to focus invasive testing on pre-filtered components and patients to reduce workload and costs of laboratory analyses (16).

Computed tomography (CT) is a commonly used imaging modality in hospitalized patients and provides non-invasive assessment of tissue morphology. Previous studies have suggested that simple attenuation measurements in CT scans correlate with hemoglobin and hematocrit levels and may be useful in predicting anemia (17-19).

By extracting a variety of mineable image features, radiomics can provide additional, higher dimensional data that can be employed to improve decision support. Current radiomic research promotes the impression that radiomic features are potentially applicable to augment data in a variety of diseases (1). However, the potential of radiomic features to assess the flowing blood compartment to predict specific components has not yet been sufficiently evaluated. The aim of this study was to assess the predictability of hemoglobin and hematocrit levels using high dimensional radiomic features in non-contrast enhanced CT scans.

\section{Methods}

\section{Patient selection}

The local Ethics committee approved this retrospective study (project number: 20-689, Goethe University Frankfurt am Main, Germany) and waived informed written consent.

A total of 181 consecutive patients (female, 54; male, 46; age, 69 (19-94) years) who underwent non-contrast dual-energy CT imaging of the thoracolumbar spine between $08 / 2018$ and $11 / 2019$ were screened for study inclusion. Inclusion criteria were (I) $>18$ years of age, (II) thoracolumbar region, (III) $1 \mathrm{~mm} 90 \mathrm{kV}$ series, (IV) hemoglobin values $\pm 24 \mathrm{~h}$ CT examination. Exclusion criteria were (I) different acquisition protocol, (II) signs of active bleeding, (III) imaging artifacts. All clinical data were obtained in clinical routine. 100 patients met the criteria and were evaluated. Figure 1 shows the flowchart of patient inclusion. Table 1 depicts patient characteristics. 
Table 1

Patients characteristics and baseline demographics

\begin{tabular}{|c|c|}
\hline Parameters & Value \\
\hline patients & 100 \\
\hline female & 54 \\
\hline male & 46 \\
\hline median age $(y)^{*}$ & $69(19-94)$ \\
\hline maximum time (h) between blood test / CT scan & \pm 24 \\
\hline mean hemoglobin $[\mathrm{mg} / \mathrm{dL}]^{\star \star}$ & $11.82(2.39,12.29,11.35)$ \\
\hline 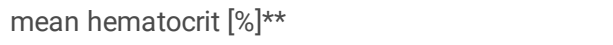 & $34.80(6.72,36.14,33.47)$ \\
\hline \multicolumn{2}{|c|}{ If not state otherwise, the numbers depict absolute numbers. } \\
\hline \multicolumn{2}{|c|}{ * Date in round parenthesis are $\mathrm{min} / \mathrm{max}$ values (interquartile range). } \\
\hline \multicolumn{2}{|c|}{$\star *$ Date in round parenthesis are standard deviation and $+/-95 \%$ confidence interva } \\
\hline $\mathrm{CT}$, computed tomography; h, hours; $y$, years & \\
\hline
\end{tabular}

\section{CT acquisition protocol}

Examinations were performed using a third-generation, dual-source, dual-energy CT system (Somatom Force; Siemens Healthineers, Forchheim, Germany). The non-contrast acquisition protocol operated the x-ray tubes at different kilovoltage settings (tube A, 90kVp, 260.9 $\pm 88 \mathrm{mAs}$, reference $300 \mathrm{mAs}$; tube B, Sn150kVp [0.64mm tin filter], $157.8 \pm 41.9 \mathrm{mAs}$, reference $188 \mathrm{mAs}$ ). The dual-energy protocol (rotation time, $0.5 \mathrm{sec} ;$ pitch, 0.6 ; collimation, $2 \times 192 \times 0.6$ ) included automatic attenuation-based tube current modulation (CARE Dose 4D; Siemens Healthineers) with a mean volume CT dose index of $12.2 \pm 3.8 \mathrm{mGy}$ and a mean dose-length product of $507.4 \pm 255.8 \mathrm{mGy} \times \mathrm{cm}$.

\section{Image reconstruction}

We applied the $90 \mathrm{kVp}$ images as they are reconstructed using isotropic voxels in clinical routine (axial, section thickness $1 \mathrm{~mm}$ and increment of $1 \mathrm{~mm}$ ) with a dedicated dual-energy medium-soft convolution kernel (Qr40, advanced model-based iterative reconstruction [ADMIRE] level of 3). For the consecutive quantitative analysis, the image stack was extracted in Digital Imaging and Communications in Medicine (DICOM) format.

\section{Radiomic analysis}

The 3D Slicer software platform (http://slicer.org, version 4.9.0) was applied to visualize and process the DICOM image stack (2, 20). For segmentation, a radiologist (SM) with two years of experience manually defined a spheric volume of interest ( $\mathrm{VOI}, 1.0 \mathrm{~cm}$ diameter) centrically in the aorta of the thoracolumbar region, sparing the aortic wall and visual artifacts (Fig. 2). All VOls were reviewed by a second radiologist (SB, two years of experience). Both radiologists were blinded to the laboratory results. Prior to feature extraction we did not perform further image manipulation as the Imaging Biomarker Standardization (IBSI) does currently not cover image preprocessing and we did perform our analysis on isotropic $1 \mathrm{~mm} \times 1 \mathrm{~mm}$ voxels (21). The open-source package PyRadiomics was used as extension within 3D Slicer to extract the radiomic features $(2,20,22)$. We extracted all standard features from seven feature classes: First Order Statistics, Shape-based, Gray Level Co-occurrence Matrix (GLCM), Gray Level Run Length Matrix (GLRLM), Gray Level Size Zone Matrix (GLSZM), Gray Level Dependence Matrix (GLDM), Neighbouring Gray Tone Difference Matrix (NGTDM), obtaining 105 features / VOI (http://pyradiomics.readthedocs.io) (22). PyRadiomics was operated using the default settings (bin width 25, enforced symmetrical GLCM, http://pyradiomics.readthedocs.io) $(22,23)$. As we used a spherical $1 \mathrm{~cm}$ VOI for segmentation, shape features were excluded for analysis, obtaining 93 features, further referred to as "all features" for statistical analysis.

\section{Statistical analysis and machine learning}

We performed radiomic feature reduction and selection to match hemoglobin [g/dL] and hematocrit [\%] values. Correlation analysis of all features was performed against hemoglobin and hematocrit values (24). We ranked the features according to the obtained p-value of the correlation analysis. The lower the p-value, the higher the ranking. Next, we used inter-correlation analysis of the features which showed significant correlation for both hemoglobin and hematocrit levels to test for collinearity (1). Features with a collinearity of $r<0.5$ were selected for further analysis. Next, we analyzed the obtained radiomic features set to differentiate moderate-to-severe anemic state. Moderate-to-severe anemia is defined by a cut-off value of hemoglobin $\leq 10-11 \mathrm{~g} / \mathrm{dL}$ depending on age and gender (25-28). For our primarily methodologically driven study we aimed to choose a uniform definition of moderate-to-severe anemia and therefore defined a cut-off value of hemoglobin $\leq 10 \mathrm{~g} / \mathrm{dL}$ for our cohort as previously proposed. We built two machine learning models based on random forest (RF) algorithms to predict moderate-to-severe anemia. The predictive power was assessed by receiver operating characteristics (ROC) curves with 100 fold cross-validation. Each run randomly drew $70 \%$ of the samples for training and tested the model with the remaining independent $30 \%$ of the data. We obtained the area under the curve (AUC), precision and accuracy. To analyze the variation of predictive power we applied a two-tailed student's $t$ test of the 100 fold cross-validated measurements. Machine learning algorithms and visualization of the decision tree were conducted in Python 3.7 using the open-source scikit-learn 0.21 .3 packages RandomForestClassifier (n_estimators = 100, max_depth = 1/(2) for one/(two) feature(s)) for RF analysis with prior normalization of features employing StandardScaler (https://scikit-learn.org/) and DecisionTreeClassifier with criterion = gini and max_depth equivalent 
to the RF-analysis (29). Further statistical analyses were performed using Prism 6.0 (GraphPad software) and JMP 14 (SAS, Cary, U.S.A.). The significance values were indicated as followed: ${ }^{*} p<0.05 ;{ }^{* *} p<0.01 ; * \star * ~ p<0.001$. The respective table and figure legends give detailed information about the statistical tests.

\section{Results}

From all radiomic features, 9 features revealed significant correlation $(p<0.001-p=0.032)$ to hemoglobin and hematocrit levels with Median ( $<0.001)$ as the highest ranked feature (Table 2). The features were found to be part of one feature class, the first-order statistics (Table 2). Grey Level Non Uniformity, a feature of the GLSZM feature class, showed correlation to hematocrit levels, but no significance to hemoglobin levels (Table 2). It was therefore excluded for further analysis.

Table 2

Top 20 radiomic features with highest variable importance based on measurement of correlations with hemoglobin and hematocrit values

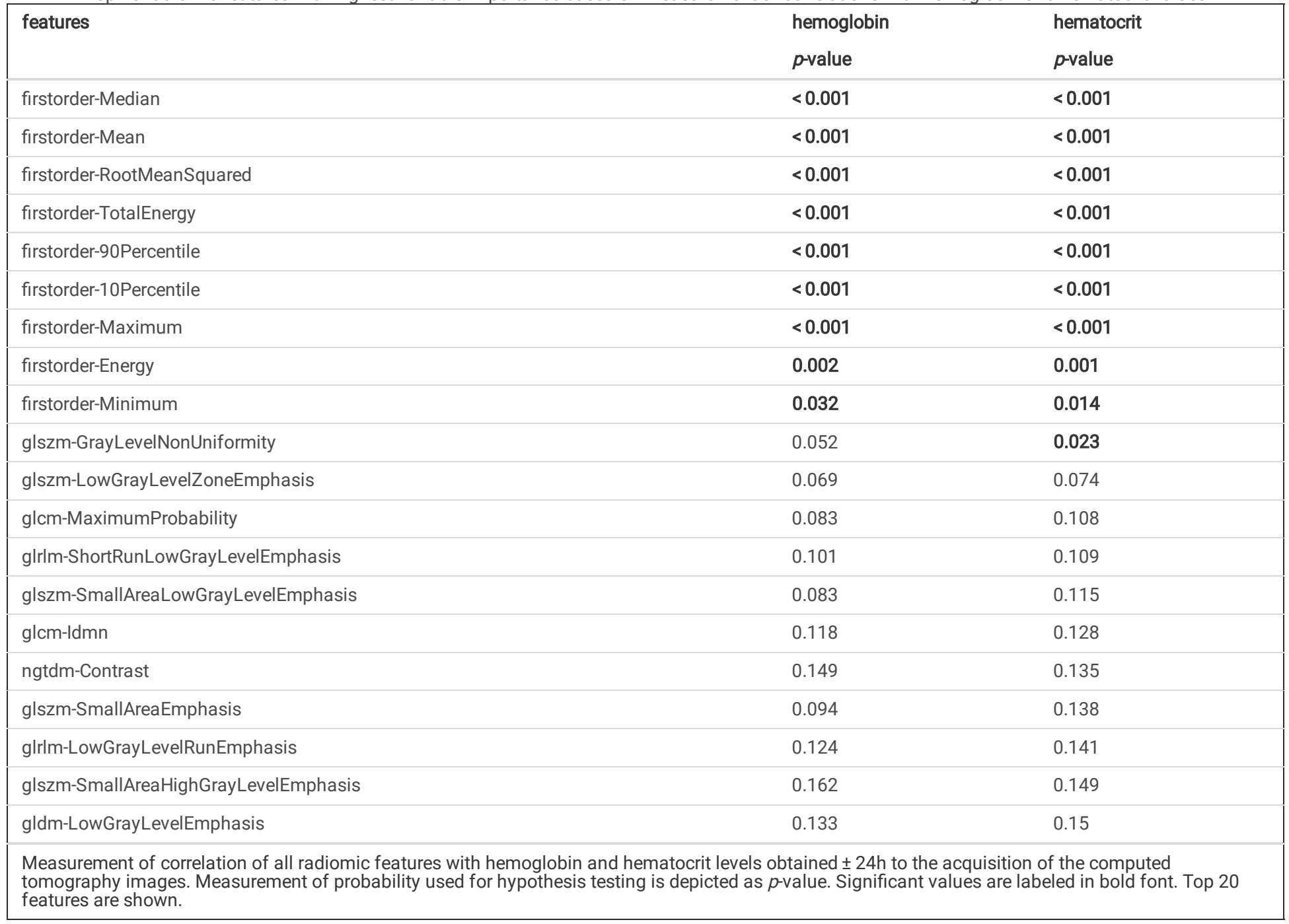

The selected features showed a high degree of collinearity (Fig. 3A, Table 3). Energy ( $r=0.387)$, Maximum $(r=0.411)$ and Minimum ( $r=0.437)$ were found to be the least correlated features to Median (Table 3). As Maximum revealed collinearity with Energy $(r=0.568)$ it was excluded for further analysis. We therefore obtained the top 3 features to correlate with hemoglobin and hematocrit levels: Median $(p<0.001$, Fig. 3B), Energy $(p=0.002$, Fig. 3C) and Minimum $(p=0.032$, Fig. 3D). 
Table 3

Matrix of correlations of radiomic features with significant correlation with hemoglobin and hematocrit levels

\begin{tabular}{|c|c|c|c|c|c|c|c|c|c|}
\hline & $\begin{array}{l}\text { firstorder- } \\
\text { Median }\end{array}$ & $\begin{array}{l}\text { firstorder- } \\
\text { Energy }\end{array}$ & $\begin{array}{l}\text { firstorder- } \\
\text { TotalEnergy }\end{array}$ & $\begin{array}{l}\text { firstorder- } \\
\text { Maximum }\end{array}$ & $\begin{array}{l}\text { firstorder- } \\
\text { RootMeanSquared }\end{array}$ & $\begin{array}{l}\text { firstorder- } \\
\text { 90Percentile }\end{array}$ & $\begin{array}{l}\text { firstorder- } \\
\text { Minimum }\end{array}$ & $\begin{array}{l}\text { firstorder- } \\
\text { 10Percentile }\end{array}$ & $\begin{array}{l}\text { firstorder- } \\
\text { Mean }\end{array}$ \\
\hline firstorder-Median & 1.000 & 0.387 & 0.971 & 0.411 & 0.977 & 0.891 & 0.437 & 0.869 & 0.993 \\
\hline firstorder-Energy & 0.387 & 1.000 & 0.422 & 0.568 & 0.427 & 0.431 & -0.139 & 0.253 & 0.388 \\
\hline $\begin{array}{l}\text { firstorder- } \\
\text { TotalEnergy }\end{array}$ & 0.971 & 0.422 & 1.000 & 0.525 & 0.992 & 0.947 & 0.339 & 0.783 & 0.973 \\
\hline $\begin{array}{l}\text { firstorder- } \\
\text { Maximum }\end{array}$ & 0.411 & 0.568 & 0.525 & 1.000 & 0.541 & 0.646 & -0.273 & 0.116 & 0.422 \\
\hline $\begin{array}{l}\text { firstorder- } \\
\text { RootMeanSquared }\end{array}$ & 0.977 & 0.427 & 0.992 & 0.541 & 1.000 & 0.961 & 0.334 & 0.781 & 0.980 \\
\hline $\begin{array}{l}\text { firstorder- } \\
\text { 90Percentile }\end{array}$ & 0.891 & 0.431 & 0.947 & 0.646 & 0.961 & 1.000 & 0.186 & 0.598 & 0.894 \\
\hline $\begin{array}{l}\text { firstorder- } \\
\text { Minimum }\end{array}$ & 0.437 & -0.139 & 0.339 & -0.273 & -0.334 & 0.186 & 1.000 & 0.665 & 0.468 \\
\hline $\begin{array}{l}\text { firstorder- } \\
\text { 10Percentile }\end{array}$ & 0.869 & 0.253 & 0.783 & 0.116 & 0.781 & 0.598 & 0.665 & 1.000 & 0.887 \\
\hline firstorder-Mean & 0.993 & 0.388 & 0.973 & 0.422 & 0.980 & 0.894 & 0.468 & 0.887 & 1.000 \\
\hline
\end{tabular}

Radiomic analysis of intraaortic blood to differentiate a threshold of hemoglobin level of $10 \mathrm{mg} / \mathrm{dL}$ revealed significant difference in the radiomic features Median ( $p<0.001$, Fig. 4A) and Minimum ( $p=0.003$, Fig. 4B) whereas Energy did not reach the level of significance $(p=0.09$, Fig. 4C) and was therefore excluded for the consecutive machine learning model development.

A random forest based, 100 fold cross-validated machine learning approach was conducted applying either Median and Minimum features (Fig. 5A, AUC $0.88 \pm 0.07$ ) or Median feature only (Fig. 5B, AUC $0.90 \pm 0.06$ ) for model building. Application of the single radiomic feature Median was superior to its combination with the feature Minimum with regard to AUC and precision measurements whereas no difference was found with regard to model accuracy (Fig. 5 C, accuracy $p=0.612, A U C p=0.015$, precision $p=0.017$ ).

We obtained a decision tree based on the radiomic feature Median (Fig. 5D). With a cutoff value of $\leq 36.5$ Hounsfield Units (HU) in an independent train/test set of patients drawn at random, we obtained a test accuracy of 0.90 and precision of 0.80 to predict moderate-to-severe anemic state.

\section{Discussion}

In this study, we examined the potential of high dimensional radiomic features to assess components of the moving blood compartment. We assumed that hemoglobin and hematocrit may be the most promising and easily non-invasively accessible values and may inherit the clinical potential to predict moderate-to-severe anemia. Examining 100 non-enhanced CT scans, we demonstrated correlation of first-order radiomic features with hemoglobin and hematocrit levels. We could obtain a cut-off value of $\leq 36.5 \mathrm{HU}$ for Median to predict moderate-to-severe anemia with an accuracy of 0.90 and a precision of 0.80 . We could show that higher dimensional radiomic features did not augment simple first order radiomic features. Based on our findings, we conclude that besides its benefit to evaluate solid tissue and tumor characteristics non-invasively, the application of higher dimensional radiomic features to analyze flowing structures such as the blood system does not seem to be promising.

Our results regarding first order radiomic features are in accordance with previous studies investigating the potential of quantitative measurements of CT density to differentiate between anemic and non-anemic conditions $(27,30,31)$. In a study of 102 patients undergoing thoracic CT scans, the authors obtained mean attenuation measurements in the left ventricle which performed better than subjective reviewer analysis (27). Another study revealed a correlation between mean attenuation values of the thoracic aorta and hemoglobin values (30). Nevertheless, these studies did not include higher dimensional radiomic features, limiting their quantitative assessment to the mean value only (30).

Quantitative imaging data have been increasingly applied in the last years. Especially in cancer research, radiomics is a rapidly evolving research field (32, 33). In contrast to results obtained from research of specific tissues or tumor types, our data suggest that the application of high dimensional radiomic features may not yield diagnostic value assessing flowing structures, such as specific components of the blood stream. In our study, high dimensional radiomic features were inferior to simple first order statistic values to estimate hemoglobin or hematocrit values and they were not applicable to predict moderate-to-severe anemia. However, first-order histogram features did significantly correlate with hemoglobin and hematocrit values with promising predictive power of therapeutically relevant anemic state.

Potential problems at each step of the radiomics workflow including image acquisition, image reconstruction, segmentation and pre-processing have already been described in literature (34). In their study from 2020, Fornacon-Wood at al. argued that different acquisition protocols (35), image reconstruction algorithms, reconstruction parameters (kernel) (36) and number of grey levels used to discretize histogram (37) affect feature values and feature 
reproducibility. Our study suggests that these issues seem to be more relevant in moving and dynamic compartments as high dimensional radiomic features had no diagnostic power for the prediction of hemoglobin and hematocrit levels. This raises the question whether most of the measured texture in a noncontrast-enhanced CT blood pool may be the effect of imaging artifacts due to the laminar flow of the blood system rather than true data of biological components.

Our study has limitations that warrant discussion. Analyzing retrospective data with continuous patient enrollment, we cannot rule out a selection bias. We had a moderate bias towards females and the older population and cannot rule out that a more balanced study population might have altered the results. Depending on age and gender, moderate-to-severe anemia is defined by a cut-off value of hemoglobin $\leq 10-11 \mathrm{~g} / \mathrm{dL}$ (25-28). As previously described, we chose a uniform cut-off value of hemoglobin $\leq 10 \mathrm{~g} / \mathrm{dL}$ for our primarily methodologically driven study but we cannot rule out that age, gender or pregnancy adjusted values might have altered the results. Our study design was limited to 100 patients and a bigger cohort might have been favorable. This bias might reduce generalizability of the results and the finally obtained cut-off value of $36.5 \mathrm{HU}$ to differentiate moderate-to-severe anemic state. We restricted the patient inclusion to one dual-energy CT scanner to exclude inter-scanner variability and to include only reconstructions with $1 \mathrm{~mm}$ isotropic voxels, nevertheless, intra-scanner variability may have occurred. We limited the region of VOI definition to the thoracolumbar region to have an adequate diameter of the aorta for VOI placement and to limit pulsation artifacts that might be present at the ascending thoracic aorta.

\section{Conclusions}

CT is a commonly applied imaging modality for a multitude of diagnostic purposes and attenuation measurements of various degrees of complexity are easily performed. We obtained simple histogram and high dimensional radiomic features and could demonstrate that histogram radiomic features enable an accurate differentiation of moderate-to-severe anaemic state and non-anemic state employing non-enhanced CT scans. We emphasize that our results are the first to show that high dimensional radiomic features are inferior to simple histogram features and do not yield additional information for the assessment of components of flowing blood in our use case to assess hemoglobin and hematocrit levels. Based on our findings, we conclude that higher dimensional radiomic features do not seem to be useful to predict components of flowing structures, probably as potential difficulties at each step of the radiomics workflow may be more relevant in flowing components. The application of radiomics may be limited to the assessment of solid tissues and tumor characteristics.

\section{Abbreviations}

AUC Area under the curve

CT Computed tomography

GLCM Gray Level Co-occurrence Matrix

GLDM Gray Level Dependence Matrix

GLRLM Gray Level Run Length Matrix

GLSZM Gray Level Size Zone Matrix

HU Hounsfield Units

NGTDM Neighboring Gray Tone Difference Matrix

ROC Receiver operating characteristics

VOI Volume of interest

\section{Declarations}

Ethics approval and consent to participate

The local Ethics committee approved this retrospective study (project number: 20-689, Goethe University Frankfurt am Main, Germany) and waived informed written consent. All methods were carried out in accordance with relevant guidelines and regulations.

\section{Consent for publication}

Not applicable.

Availability of data and materials

The datasets used and/or analysed during the current study are available from the corresponding author on reasonable request.

\section{Competing interests}

The authors declare that they have no competing interests. 
This study was not supported by any funding.

Authors' contribution

SM, SB: Conceptualization, Data curation, Formal analysis, Investigation, Methodology, Project administration, Software, Supervision, Validation, Visualization, Writing - original draft, Writing - review \& editing

TJV: Data curation, Formal analysis, Investigation, Methodology, Project administration, Resources, Software, Supervision, Validation, Visualization, Writing review \& editing

SSM, MA, LL: Formal analysis, Methodology, Project administration, Software, Supervision, Validation, Writing - review \& editing

JA, YZ, IK: Data curation, Formal analysis, Methodology, Software, Validation, Visualization, Writing - review \& editing

Acknowledgments

Not applicable.

\section{References}

1. Gillies RJ, Kinahan PE, Hricak H. Radiomics: Images Are More than Pictures, They Are Data. Radiology [Internet]. 2016 Feb [cited 2020 Feb 20];278(2):563-77. Available from: http://www.ncbi.nlm.nih.gov/pubmed/26579733

2. Kumar V, Gu Y, Basu S, Berglund A, Eschrich SA, Schabath MB, et al. Radiomics: the process and the challenges. Magn Reson Imaging [Internet]. 2012 Nov [cited 2020 Feb 17];30(9):1234-48. Available from: http://www.ncbi.nlm.nih.gov/pubmed/22898692

3. Lambin P, Rios-Velazquez E, Leijenaar R, Carvalho S, van Stiphout RGPM, Granton P, et al. Radiomics: extracting more information from medical images using advanced feature analysis. Eur J Cancer [Internet]. 2012 Mar [cited 2020 Feb 17];48(4):441-6. Available from: http://www.ncbi.nlm.nih.gov/pubmed/22257792

4. Bi WL, Hosny A, Schabath MB, Giger ML, Birkbak NJ, Mehrtash A, et al. Artificial intelligence in cancer imaging: Clinical challenges and applications. CA Cancer J Clin [Internet]. 2019 Feb 5 [cited 2020 Feb 20];69(2):caac.21552. Available from: http://www.ncbi.nlm.nih.gov/pubmed/30720861

5. Aerts HJWL, Velazquez ER, Leijenaar RTH, Parmar C, Grossmann P, Carvalho S, et al. Decoding tumour phenotype by noninvasive imaging using a quantitative radiomics approach. Nat Commun [Internet]. 2014 Jun 3 [cited 2020 Feb 20];5:4006. Available from:

http://www.ncbi.nlm.nih.gov/pubmed/24892406

6. Parmar C, Leijenaar RTH, Grossmann P, Rios Velazquez E, Bussink J, Rietveld D, et al. Radiomic feature clusters and prognostic signatures specific for Lung and Head \&amp; Neck cancer. Sci Rep [Internet]. 2015 Jun 5 [cited 2020 Feb 20];5:11044. Available from:

http://www.ncbi.nlm.nih.gov/pubmed/26251068

7. Liu Z, Wang S, Dong D, Wei J, Fang C, Zhou X, et al. The Applications of Radiomics in Precision Diagnosis and Treatment of Oncology: Opportunities and Challenges. Theranostics [Internet]. 2019 [cited 2020 Feb 20];9(5):1303-22. Available from: http://www.ncbi.nlm.nih.gov/pubmed/30867832

8. Turri G, Pedrazzani C, Malerba G, Gecchele G, Conti C, Ruzzenente A, et al. Effect of peri-operative blood transfusions on long-term prognosis of patients with colorectal cancer. Blood Transfus [Internet]. 2020 Dec 22 [cited 2021 Feb 8]; Available from: http://www.ncbi.nlm.nih.gov/pubmed/33370231

9. Mansour D, Hofmann A, Gemzell-Danielsson K. A Review of Clinical Guidelines on the Management of Iron Deficiency and Iron-Deficiency Anemia in Women with Heavy Menstrual Bleeding. Adv Ther [Internet]. 2021 Jan [cited 2021 Feb 8];38(1):201-25. Available from:

http://www.ncbi.nlm.nih.gov/pubmed/33247314

10. Adel A, Awada W, Abdelhamid B, Omar H, Abd El Dayem O, Hasanin A, et al. Accuracy and trending of non-invasive hemoglobin measurement during different volume and perfusion statuses. J Clin Monit Comput [Internet]. 2018 Dec 15 [cited 2020 Feb 16];32(6):1025-31. Available from: http://link.springer.com/10.1007/s10877-018-0101-z

11. Bruns B, Lindsey M, Rowe K, Brown S, Minei JP, Gentilello LM, et al. Hemoglobin drops within minutes of injuries and predicts need for an intervention to stop hemorrhage. J Trauma [Internet]. 2007 Aug [cited 2020 Feb 16];63(2):312-5. Available from: https://insights.ovid.com/crossref?an=00005373200708000-00010

12. McLean E, Cogswell M, Egli I, Wojdyla D, de Benoist B. Worldwide prevalence of anaemia, WHO Vitamin and Mineral Nutrition Information System, 19932005. Public Health Nutr [Internet]. 2009 Apr 23 [cited 2020 Feb 17];12(04):444. Available from: http://www.ncbi.nlm.nih.gov/pubmed/18498676

13. Guilbert J. The World Health Report 2002 - Reducing Risks, Promoting Healthy Life. Educ Heal Chang Learn Pract [Internet]. 2003 Jan 1 [cited 2020 Feb 17];16(2):230-230. Available from: http://www.ncbi.nlm.nih.gov/pubmed/14741909

14. Zwart A, van Assendelft OW, Bull BS, England JM, Lewis SM, Zijlstra WG. Recommendations for reference method for haemoglobinometry in human blood (ICSH standard 1995) and specifications for international haemiglobinocyanide standard (4th edition). J Clin Pathol [Internet]. 1996 Apr 1 [cited 2020 Feb 16];49(4):271-4. Available from: http://jcp.bmj.com/cgi/doi/10.1136/jcp.49.4.271

15. Dychter SS, Gold DA, Carson D, Haller M. Intravenous Therapy. J Infus Nurs [Internet]. 2012 [cited 2020 Feb 16];35(2):84-91. Available from: http://www.ncbi.nlm.nih.gov/pubmed/22382792 
16. Meybohm P, Richards T, Isbister J, Hofmann A, Shander A, Goodnough LT, et al. Patient Blood Management Bundles to Facilitate Implementation. Transfus Med Rev [Internet]. 2017 [cited 2020 Feb 21];31(1):62-71. Available from: http://www.ncbi.nlm.nih.gov/pubmed/27317382

17. New PFJ, Aronow S. Attenuation Measurements of Whole Blood and Blood Fractions in Computed Tomography. Radiology [Internet]. 1976 Dec [cited 2020 Feb 16];121(3):635-40. Available from: http://www.ncbi.nlm.nih.gov/pubmed/981659

18. Foster M, Nolan RL, Lam M. Prediction of anemia on unenhanced computed tomography of the thorax. Can Assoc Radiol J [Internet]. 2003 Feb [cited 2020 Feb 17];54(1):26-30. Available from: http://www.ncbi.nlm.nih.gov/pubmed/12625080

19. Collins AJ, Gillespie S, Kelly BE. Can computed tomography identify patients with anaemia? Ulster Med J [Internet]. 2001 Nov [cited 2020 Feb 16];70(2):116-8. Available from: http://www.ncbi.nlm.nih.gov/pubmed/11795761

20. Fedorov A, Beichel R, Kalpathy-Cramer J, Finet J, Fillion-Robin J-C, Pujol S, et al. 3D Slicer as an image computing platform for the Quantitative Imaging Network. Magn Reson Imaging [Internet]. 2012 Nov [cited 2020 Apr 9];30(9):1323-41. Available from: http://www.ncbi.nlm.nih.gov/pubmed/22770690

21. Zwanenburg A, Leger S, Vallières M, Löck S. Image biomarker standardisation initiative. 2016 Dec 21 [cited 2020 Apr 9]; Available from: http://arxiv.org/abs/1612.07003

22. van Griethuysen JJM, Fedorov A, Parmar C, Hosny A, Aucoin N, Narayan V, et al. Computational Radiomics System to Decode the Radiographic Phenotype. Cancer Res [Internet]. 2017 Nov 1 [cited 2020 Apr 9];77(21):e104-7. Available from: http://cancerres.aacrjournals.org/lookup/doi/10.1158/0008-5472.CAN-17-0339

23. Schwier M, van Griethuysen J, Vangel MG, Pieper S, Peled S, Tempany C, et al. Repeatability of Multiparametric Prostate MRI Radiomics Features. Sci Rep [Internet]. 2019 Jul 1 [cited 2020 Apr 9];9(1):9441. Available from: http://www.nature.com/articles/s41598-019-45766-Z

24. Parmar C, Grossmann P, Bussink J, Lambin P, Aerts HJWL. Machine Learning methods for Quantitative Radiomic Biomarkers. Sci Rep [Internet]. 2015 Oct 17 [cited 2020 Apr 9];5(1):13087. Available from: http://www.nature.com/articles/srep13087

25. Kassebaum NJ, GBD 2013 Anemia Collaborators. The Global Burden of Anemia. Hematol Oncol Clin North Am [Internet]. 2016 Apr [cited 2020 Apr 9];30(2):247-308. Available from: http://www.ncbi.nlm.nih.gov/pubmed/27040955

26. World Health Organization. Haemoglobin concentrations for the diagnosis of anaemia and assessment of severity [Internet]. 2011 [cited 2021 Feb 8]. Available from: https://apps.who.int/iris/bitstream/handle/10665/85839/WHO_NMH_NHD_MNM_11.1_eng.pdf?ua=1

27. Title RS, Harper K, Nelson E, Evans T, Tello R. Observer performance in assessing anemia on thoracic CT. AJR Am J Roentgenol [Internet]. 2005 Nov [cited 2020 Mar 15];185(5):1240-4. Available from: http://www.ncbi.nlm.nih.gov/pubmed/16247142

28. Le CHH. The Prevalence of Anemia and Moderate-Severe Anemia in the US Population (NHANES 2003-2012). Collins JF, editor. PLoS One [Internet]. 2016 Nov 15 [cited 2020 Apr 5];11(11):e0166635. Available from: http://dx.plos.org/10.1371/journal.pone.0166635

29. Pedregosa F, Varoquaux G, Gramfort A, Michel V, Thirion B, Grisel O, et al. Scikit-learn: Machine Learning in Python. 2012 Jan 2 [cited 2020 Apr 9 ]; Available from: http://arxiv.org/abs/1201.0490

30. Jung C, Groth M, Bley TA, Henes FO, Treszl A, Adam G, et al. Assessment of anemia during CT pulmonary angiography. Eur J Radiol [Internet]. 2012 Dec [cited 2020 Mar 15];81(12):4196-202. Available from: http://www.ncbi.nlm.nih.gov/pubmed/22889590

31. Bruni SG, Patafio FM, Dufton JA, Nolan RL, Islam O. The assessment of anemia from attenuation values of cranial venous drainage on unenhanced computed tomography of the head. Can Assoc Radiol J [Internet]. 2013 Feb [cited 2020 Mar 15];64(1):46-50. Available from: http://www.ncbi.nlm.nih.gov/pubmed/22397828

32. Thawani R, McLane M, Beig N, Ghose S, Prasanna P, Velcheti V, et al. Radiomics and radiogenomics in lung cancer: A review for the clinician. Lung Cancer [Internet]. 2018 [cited 2020 Mar 15];115:34-41. Available from: http://www.ncbi.nlm.nih.gov/pubmed/29290259

33. Yip SSF, Aerts HJWL. Applications and limitations of radiomics. Phys Med Biol [Internet]. 2016 [cited 2020 Mar 15];61(13):R150-66. Available from: http://www.ncbi.nlm.nih.gov/pubmed/27269645

34. Fornacon-Wood I, Faivre-Finn C, O'Connor JPB, Price GJ. Radiomics as a personalized medicine tool in lung cancer: Separating the hope from the hype. Lung Cancer [Internet]. 2020 [cited 2021 Mar 28];146:197-208. Available from: http://www.ncbi.nlm.nih.gov/pubmed/32563015

35. Ger RB, Zhou S, Chi P-CM, Lee HJ, Layman RR, Jones AK, et al. Comprehensive Investigation on Controlling for CT Imaging Variabilities in Radiomics Studies. Sci Rep [Internet]. 2018 [cited 2021 Mar 28];8(1):13047. Available from: http://www.ncbi.nlm.nih.gov/pubmed/30158540

36. Lu L, Ehmke RC, Schwartz LH, Zhao B. Assessing Agreement between Radiomic Features Computed for Multiple CT Imaging Settings. PLoS One [Internet]. 2016 [cited 2021 Mar 28];11(12):e0166550. Available from: http://www.ncbi.nlm.nih.gov/pubmed/28033372

37. Shafiq-Ul-Hassan M, Zhang GG, Latifi K, Ullah G, Hunt DC, Balagurunathan Y, et al. Intrinsic dependencies of CT radiomic features on voxel size and number of gray levels. Med Phys [Internet]. 2017 Mar [cited 2021 Mar 28];44(3):1050-62. Available from:

http://www.ncbi.nlm.nih.gov/pubmed/28112418

\section{Figures}




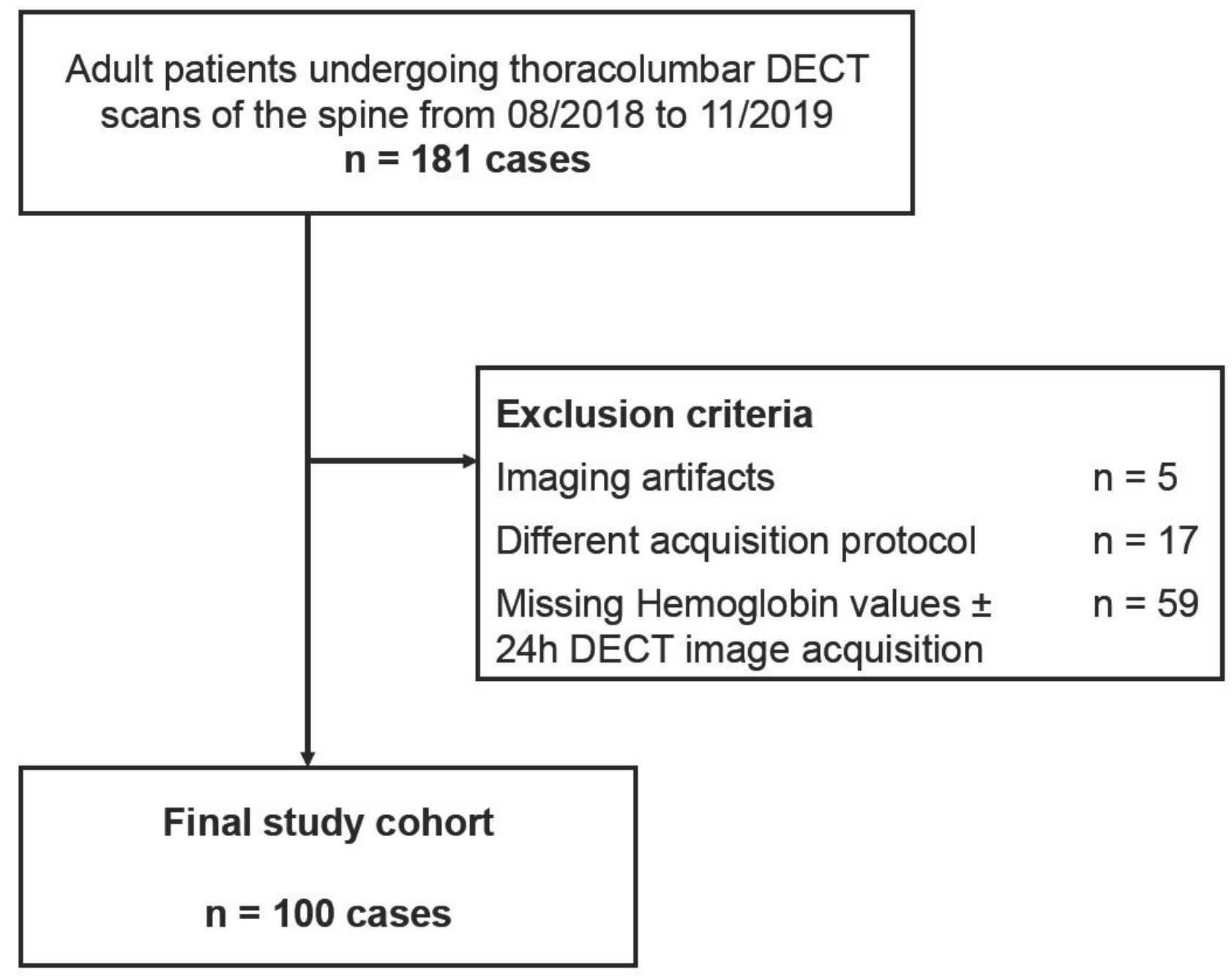

Figure 1

CLAIM flowchart of patient inclusion into the study CLAIM, Checklist for Artificial Intelligence in Medical Imaging; DECT, dual-energy computed tomography. 
A

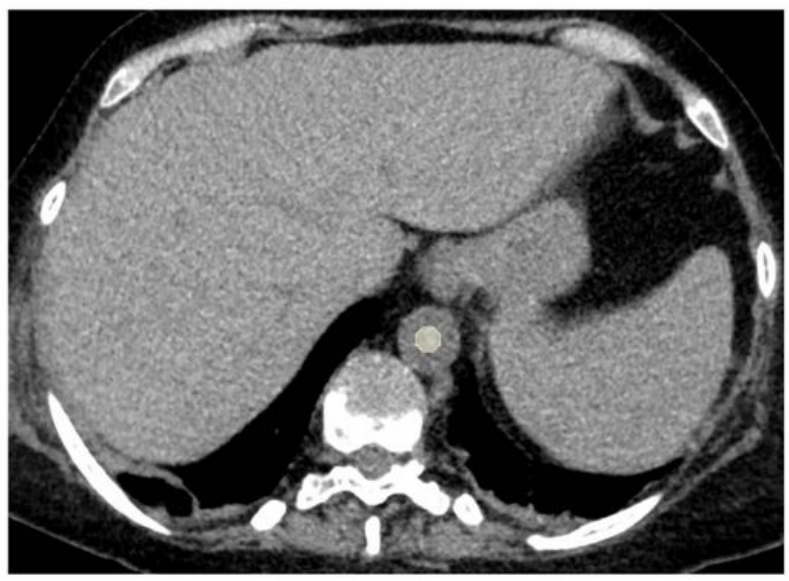

C

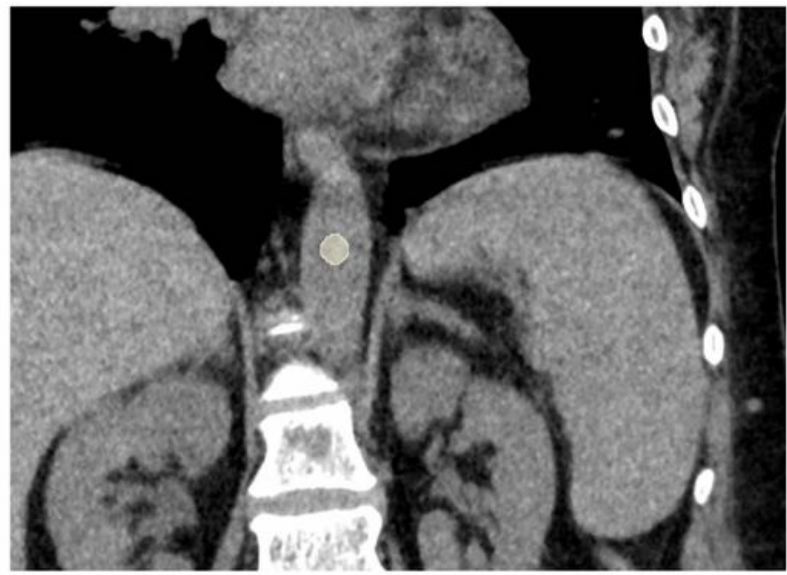

B

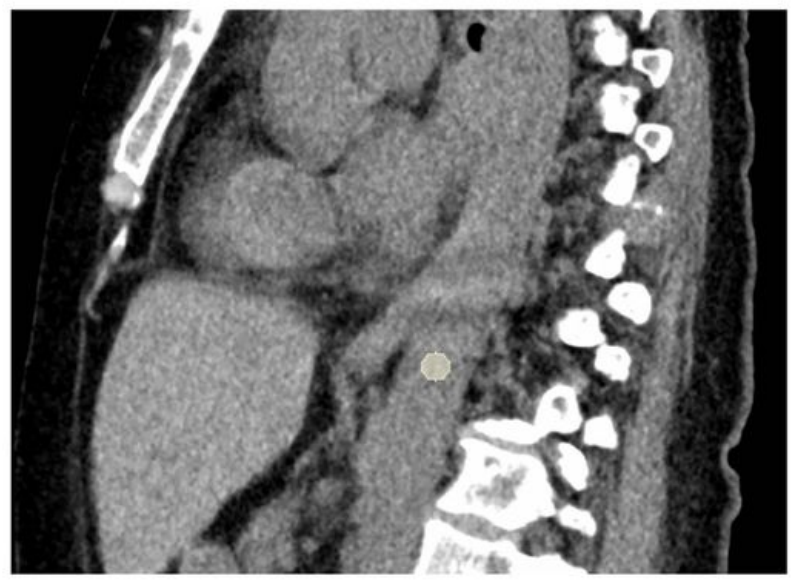

D

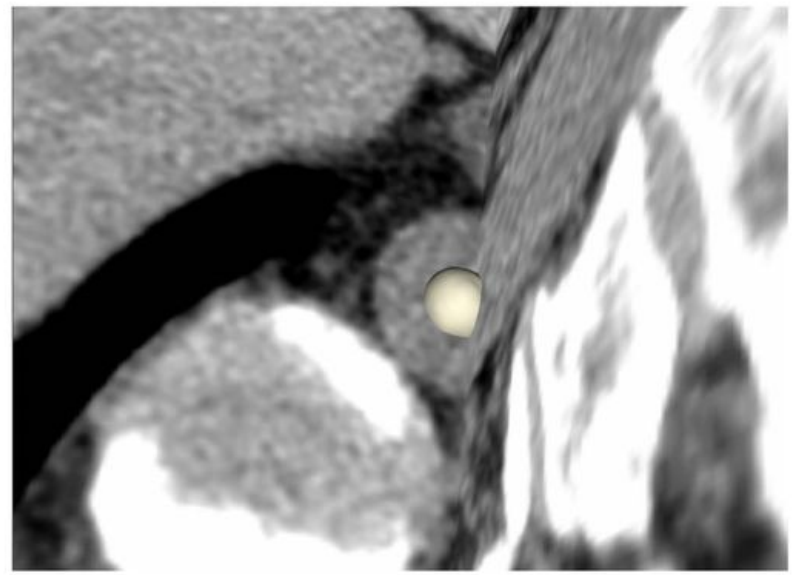

Figure 2

Representative images of the measurement technique Axial (A), sagittal (B) and coronal (C) plane with 3D-volume rendering (D) of a standard volume of interest (VOI) placement is shown in a patient with a hemoglobin and hematocrit level of $7.2 \mathrm{~g} / \mathrm{dL}$ and $22.4 \%$, respectively. A spherical VOI with $1 \mathrm{~cm}$ in diameter was placed within the lumen of the thoracoabdominal aorta as described in detail in the materials and methods section. 
A
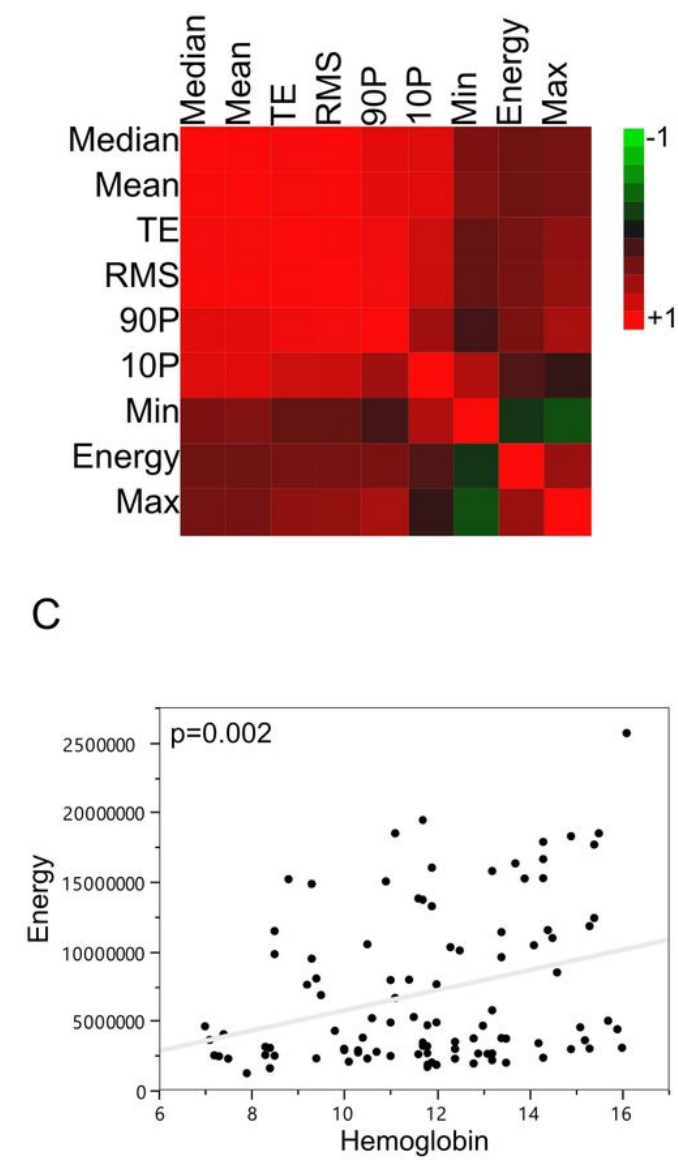

B

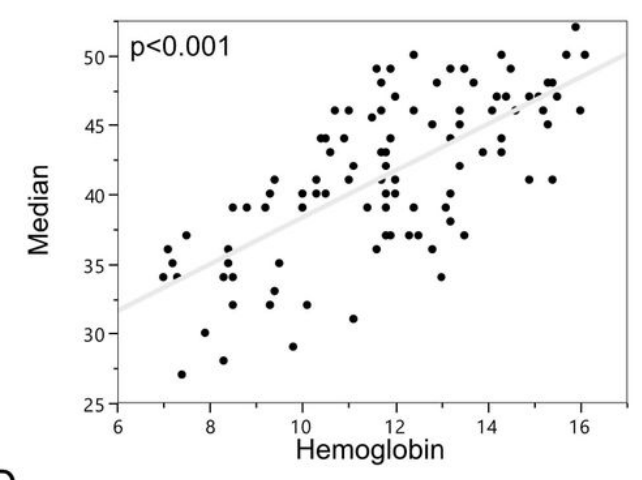

D

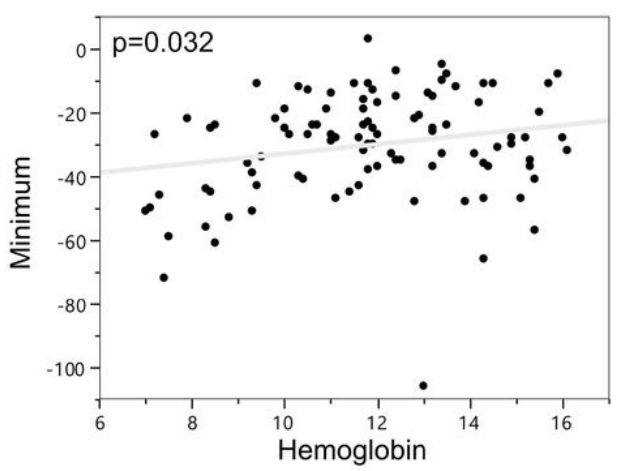

Figure 3

Analysis of radiomic features that are significantly correlated with hemoglobin and hematocrit levels The matrix of correlations of the selected radiomic features with highest correlation to the hemoglobin $[\mathrm{g} / \mathrm{dL}]$ and hematocrit [\%] levels obtained $\pm 24 \mathrm{~h}$ to computed tomography images are shown (A). Exemplary scatter plots of the correlation of hemoglobin values with the prioritized top 3 radiomic features are shown (B-D). All depicted features belong to the feature class of first-order statistics. 10P $=10$ Percentile; 90P $=90$ Percentile; Max = Maximum; Min $=$ Minimum; RMS $=$ Root Mean Squared; TE $=$ Total Energy. 
A

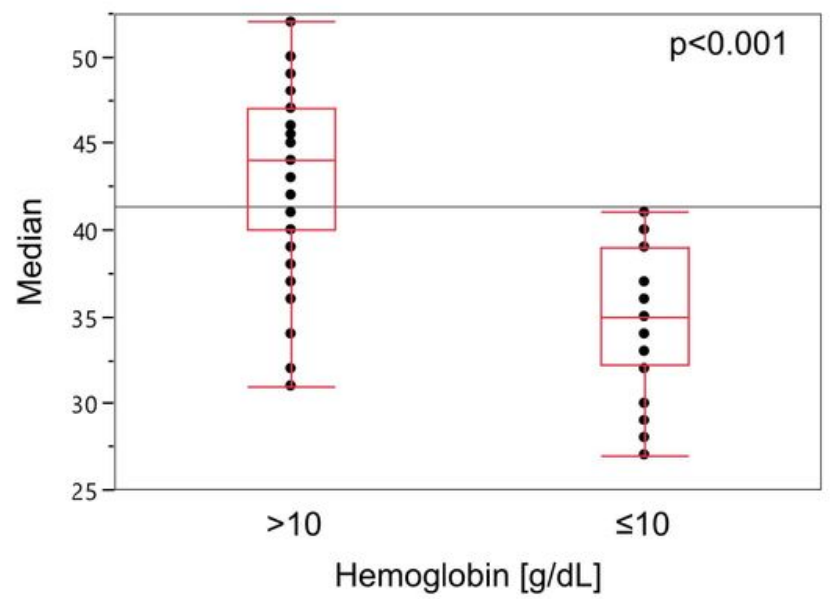

C

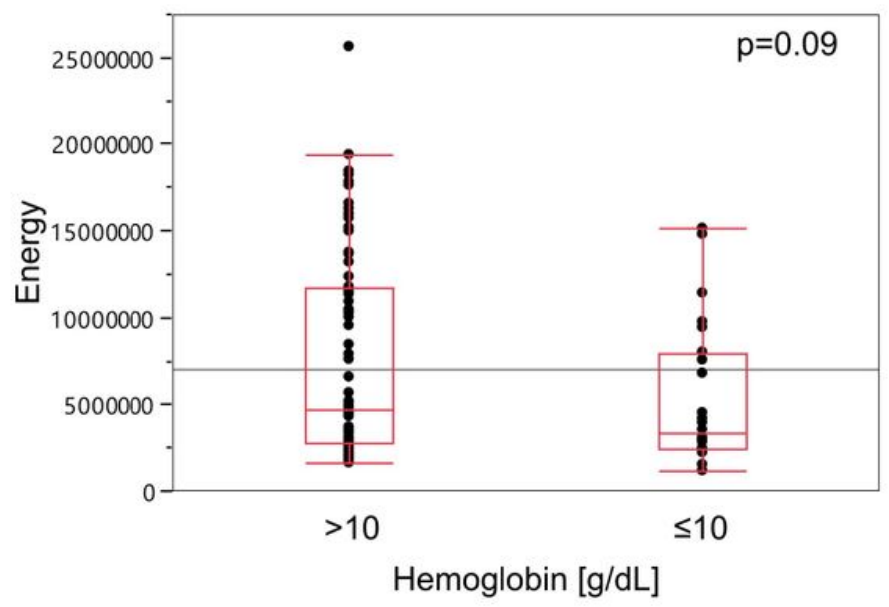

B

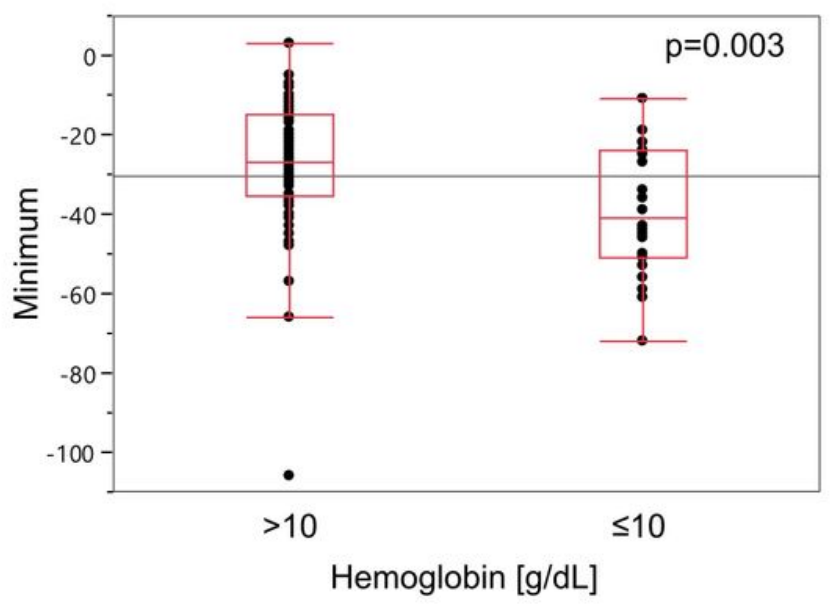

Figure 4

Radiomic features to decipher moderate-to-severe anemia Box-Whisker Plots for the radiomic features Median (A), Minimum (B) and Energy (C) versus hemoglobin levels are shown. Hemoglobin values were split according to the threshold of $10 \mathrm{~g} / \mathrm{dL}$ to differentiate moderate-to-severe anemia (25-28). Statistical analyses are depicted using two-tailed student's t-test. 
A

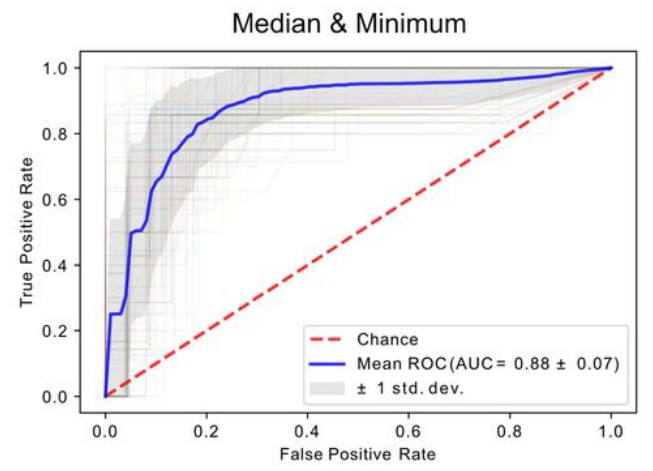

C
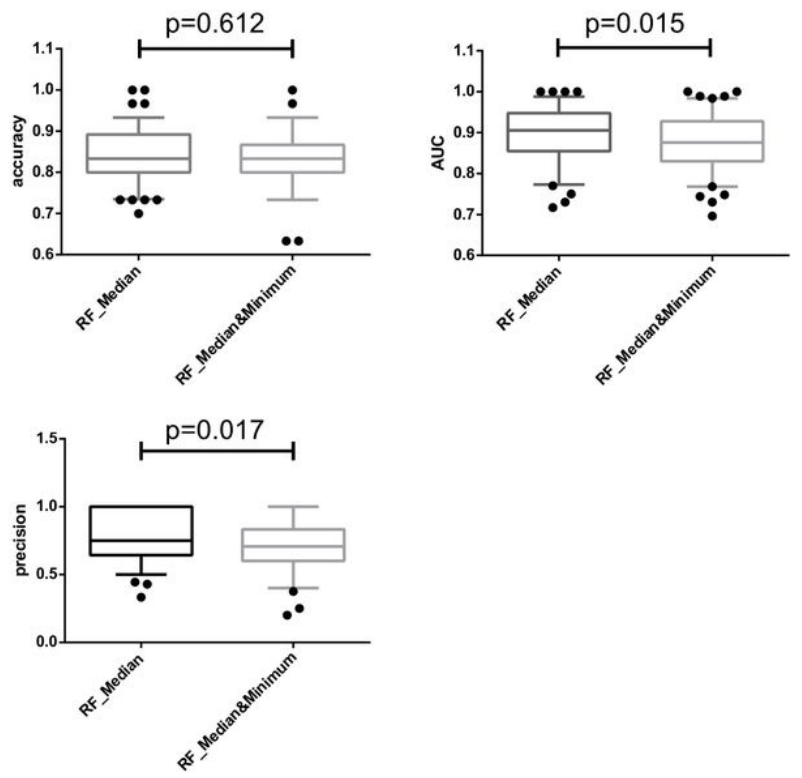

B

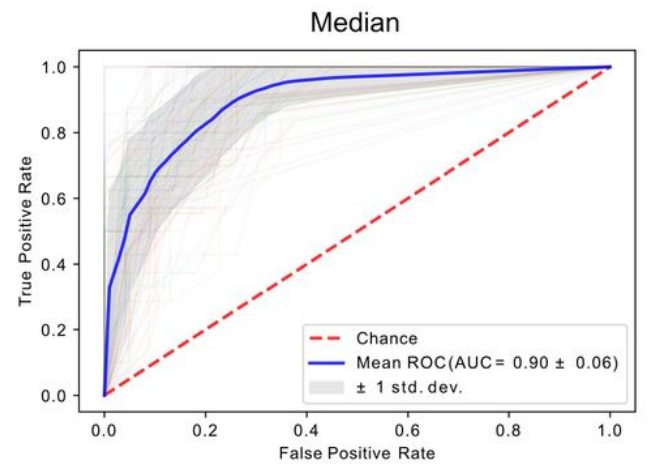

$\mathrm{D}$

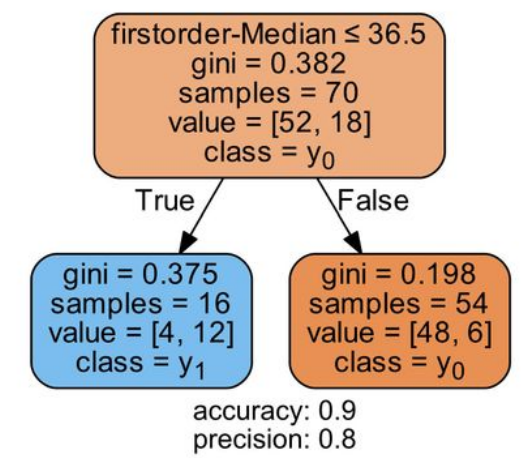

Figure 5

Median density measurement of Hounsfield units reveals the best working model to predict moderate-to-severe anemia Analysis of prediction performance for moderate-to-severe anemia with 2 variant feature subsets applying random forest (RF) machine learning algorithms (A-C). 100 fold cross-validated (colors) receiver operating characteristics (ROC) curve analysis of the validation cohort with mean ROC curve (blue) and \pm 1 standard deviation (grey area) are shown for Median and Minimum (A) or Median only (B). RF maximum depth was 2 (A) and 1 (B). C shows the Box-Whisker Plots with 5-95\% percentile for both cross-validated prediction models with the respective accuracy, area under the curve (AUC) and precision. Two-tailed, unpaired student's t-test was applied for model comparison (C, p-values). In D, an exemplary decision tree with a depth of 1 for Median is shown to stratify moderate-to-severe anemic state $(\mathrm{y} 0=\mathrm{Hb}>10 \mathrm{~g} / \mathrm{dL} ; \mathrm{y} 1=\mathrm{Hb} \leq 10 \mathrm{~g} / \mathrm{dL}$ ). The decision tree with Gini-based fitting applied a training cohort of $70 \%$ drawn at random and an independent test cohort of $30 \%$. 